\title{
Hypoxia-dependent expression of MAP17 coordinates the Warburg effect to tumor growth in hepatocellular carcinoma
}

Fangyuan Dong ${ }^{1,2,3,4 \dagger}$, Rongkun $\mathrm{Li}^{5 \dagger}$, Jiaofeng Wang ${ }^{1,2,3,4 \dagger}$, Yan Zhang ${ }^{1}$, Jianfeng Yao ${ }^{1}$, Shu-Heng Jiang ${ }^{6}$, Xiaona $\mathrm{Hu}^{1,2,3,4^{*}}$, Mingxuan Feng ${ }^{7^{*}}$ and Zhijun Bao ${ }^{1,2,3,4^{*}}$

\section{Abstract}

Background: Reprogrammed glucose metabolism, also known as the Warburg effect, which is essential for tumor progression, is regarded as a hallmark of cancer. MAP17, a small 17-kDa non-glycosylated membrane protein, is frequently dysregulated in human cancers. However, its role in hepatocellular carcinoma (HCC) remains largely unknown.

Methods: Immunohistochemistry was used to analyze the expression pattern of MAP17 in HCC. Loss-of-function and gain-of-function studies were performed to investigate the oncogenic roles of MAP17 in vitro and in vivo. RNA sequencing, co-immunoprecipitation, immunofluorescence and western blotting were used to study the molecular mechanism of MAP17 affecting the tumor growth and glycolytic phenotype of HCC.

(Continued on next page)

\footnotetext{
* Correspondence: huxnfd@hotmail.com; heartsfool@sohu.com; zhijunbao@fudan.edu.cn

${ }^{\dagger}$ Fangyuan Dong, Rongkun Li and Jiaofeng Wang contributed equally to this work.

'Department of Gastroenterology, Huadong Hospital, Shanghai Medical College, Fudan University, No.221 Yan'an West Road, Shanghai 200040, P.R. China

${ }^{7}$ Department of Liver Surgery, Ren Ji Hospital, School of Medicine, Shanghai Jiao Tong University, 1630 Dongfang Road, Shanghai 200127, P.R. China Full list of author information is available at the end of the article
}

C C The Author(s). 2021 Open Access This article is licensed under a Creative Commons Attribution 4.0 International License, which permits use, sharing, adaptation, distribution and reproduction in any medium or format, as long as you give appropriate credit to the original author(s) and the source, provide a link to the Creative Commons licence, and indicate if changes were made. The images or other third party material in this article are included in the article's Creative Commons licence, unless indicated otherwise in a credit line to the material. If material is not included in the article's Creative Commons licence and your intended use is not permitted by statutory regulation or exceeds the permitted use, you will need to obtain permission directly from the copyright holder. To view a copy of this licence, visit http://creativecommons.org/licenses/by/4.0/ The Creative Commons Public Domain Dedication waiver (http://creativecommons.org/publicdomain/zero/1.0/) applies to the data made available in this article, unless otherwise stated in a credit line to the data. 
(Continued from previous page)

Results: An integrative analysis showed that MAP17, a small 17-kDa non-glycosylated membrane protein, is significantly related to the glycolytic phenotype of hepatocellular carcinoma (HCC). Firstly, we found that MAP17 expression is hypoxia-dependent and predicts a poor prognosis in HCC. Genetic silencing of MAP17 reduced the rate of glucose uptake, lactate release, extracellular acidification rate, and expression of glycolytic genes. Ectopic expression of wild type MAP17 but not its PDZ binding domain mutant MAP17-PDZm increased tumor glycolysis. Further research showed that MAP17 knockdown markedly retarded in vivo tumor growth in HCC. Importantly, attenuation of tumor glycolysis by galactose largely hijacked the growth-promoting role of MAP17 in HCC cells. RNA sequencing analysis revealed that MAP17 knockdown leads to transcriptional changes in the ROS metabolic process, cell surface receptor signaling, cell communication, mitotic cell cycle progression, and regulation of cell differentiation. Mechanistically, MAP17 exerted an increased tumoral phenotype associated with an increase in reactive oxygen species (ROS), which activates downstream effectors AKT and HIF1a to enhance the Warburg effect. In HCC clinical samples, there is a close correlation between MAP17 expression and HIF1a or phosphorated level of AKT.

Conclusions: Our results show that MAP17 is a novel glycolytic regulator, and targeting MAP17/ROS pathway may be an alternative approach for the prevention and treatment of HCC.

Keywords: PDZK1IP1, SLC2A1, Aerobic glycolysis, Liver cancer, Hypoxia-inducible factor

\section{Introduction}

Hepatocellular carcinoma (HCC) comprises the majority of primary liver cancer and represents a serious international health problem [1]. HCC ranks as the fourth leading cause of cancer-related death globally and is expected to continue to increase in the next two decades. Despite great achievement made in diagnosis and therapy, the 5-year survival rate of HCC patients is still unsatisfactory [2]. In advanced HCC patients, the recurrence rate is approximately $80 \%$ [3]. Therefore, elucidating novel molecular mechanisms that can help determine how HCC development and progression are still urgently needed.

The hypoxic tumor microenvironment is a major character of solid tumors, including HCC. To meet the high demand for rapid cell proliferation, cancer cells reprogram their metabolisms. Reprogrammed energy metabolism is emerged as a novel hallmark of cancers, especially aerobic glycolysis, also known as the Warburg effect [4]. Aerobic glycolysis can provide a large number of intermediate products for the synthesis of nucleotides, lipids, and non-essential amino acids, and therefore support the rapid proliferation of tumor cells. As the last step of aerobic glycolysis, the produced lactic acid can acidify the tumor microenvironment, thereby inhibiting the activity of immune cells and promoting tumor invasion and metastasis. At the same time, NADPH and reduced glutathione are produced through pentose phosphate branching, which can help tumor cells avoid oxidative stress-mediated damage [5]. Thus, compared with oxidative phosphorylation in normal cells, tumor cells are more dependent on aerobic glycolysis [5]. In HCC, aerobic glycolysis is widespread and is closely related to tumor occurrence, invasion, and patient prognosis. Blocking aerobic glycolysis can significantly inhibit tumor growth and increase tumor cell sensitivity to chemotherapy drugs [6-8]. Therefore, targeting aerobic glycolysis has extremely high translational significance for the development of new therapeutics in HCC.

Previously, Hypoxia-inducible factors (HIFs, including HIF $1 \alpha$ and HIF $2 \alpha$ ) and several oncogenic proteins (AKT, FOXK1/2, SIX1, and c-MYC) are broadly expressed in human cancers and have been reported to be involved in the regulation of the Warburg effect [913]. MAP17 is a small non-glycosylated protein, localized in the cell membrane and Golgi apparatus, and is encoded by the PDZK1IP1 gene [14]. The hydrophobic $\mathrm{N}$-terminus of MAP17 consists of 13 amino acids and contains a PDZ binding domain and two transmembrane regions. MAP17 can interact with proteins containing PDZ domains such as PDZK1 (NHRF3) and other NHRF proteins (NaPiIla and NHe3). Through interaction with NHRF3 and NHRF4, MAP17 can lead to the internalization of NaPilla in the trans-Golgi network [15]. By stimulating sodium-dependent glucose transporter (SGLT1/2), MAP17 can increase the sodium-dependent specific transport of mannose and glucose in Xenopus oocytes [16]. In tumors, most studies have shown that MAP17 acts as an oncogene with increased tumorigenicity when it is overexpressed [17-21]. However, the cellular function and molecular mechanism of MAP17 in HCC remain scanty.

In this study, we performed an integrated analysis and identified MAP17 as a hypoxia-induced glycolytic regulator in HCC. By loss-of-function and gain-of-function studies, we revealed that MAP17 couples aerobic glycolysis to tumor growth in HCC via activation of ROS/ AKT pathway. Thus, this study indicates that MAP17- 
mediated cascades may act as a candidate therapeutic target for HCC treatment.

\section{Materials and methods Cell culture}

Liver cancer cell lines (SK-H929, HepG2, SMMC-7721, Huh-7, MHCC-97H, and HCC-LM3) used in this study were acquired from the Cell Bank of the Chinese Academy of Sciences (Shanghai, China). Mycoplasma contamination detection and short tandem repeat (STR) profiling were performed before cell experiments. Cells were maintained in a $37^{\circ} \mathrm{C}$ humidified incubator with $5 \% \mathrm{CO}_{2}$ and cultured in Dulbecco's modified Eagle's medium (Gibco, 11,965,092) or Roswell Park Memorial Institute 1640 medium (Gibco, 11,875,093), supplemented with $1 \%(\mathrm{v} / \mathrm{v})$ streptomycin-penicillin (Sigma-Aldrich, Shanghai, China) and 10\% fetal bovine serum (FBS, Gibco). To mimic hypoxic conditions, HCC cells were maintained in a low-oxygen chamber $\left(<1 \% \mathrm{O}_{2}\right)$ for $24 \mathrm{~h}$. The antioxidant $\mathrm{N}$-acetylcysteine (NAC), galactose, MG132, and AKT inhibitor MK-2206 were all purchased from Selleck (Shanghai, China). CHX was purchased from Sigma-Aldrich (Shanghai, China).

\section{Online database analysis}

The BioGPS database (http://biogps.org/) was used to determine the expression pattern of MAP17 in normal human tissues. The Gene Expression Profiling Interactive Analysis 2 (GEPIA2) database (http://gepia. cancer-pku.cn/index.html) [22] was used to determine the expression landscape of MAP17 across 33 types of human cancers.

\section{Clinical samples}

All HCC tissue samples used in this study were obtained from the Department of Liver Surgery, Ren Ji Hospital, School of Medicine, Shanghai Jiaotong University. An HCC tissue microarray containing 202 matched tumor and nontumor tissues was generated as reported previously [23]. All clinical specimens were obtained with informed consent, and protocols were approved by the ethical review committee of the Ren Ji Hospital, School of Medicine, Shanghai Jiaotong University.

\section{Immunohistochemistry}

Immunohistochemical (IHC) analysis was performed as described previously [24]. The primary antibodies used for IHC analysis were shown as follows: MAP17 (1:200, Abcam, ab156014), p-AKT (1:100, Cell Signaling Technology, \#4060), and Ki67 (1:400, Cell Signaling Technology, \#9449). Scoring was evaluated independently by two senior pathologists who were blinded to clinicopathologic data and calculated based on the percentage of positive staining cells and staining intensity.

\section{Generation of stably knockdown cells}

Short hairpin RNAs (shRNAs) against MAP17 gene were transfected along with a three plasmid system (pPACKH1-REV, pPACKH1-GAG, and pVSV-G) into HEK293T cells using Lipofectamine 2000 (Invitrogen, Carlsbad, CA, USA) according to the manufacturer's protocols. Conditioned medium $(\mathrm{CM})$ containing retroviral particles was collected at $48 \mathrm{~h}$ after transfection, and filtered through $0.45-\mu \mathrm{m}$ filters. After incubating with conditioned medium containing retroviral particles and $8 \mathrm{mg} / \mathrm{ml}$ polybrene (Sigma-Aldrich, H9268, St. Louis, MO) for two days, the target cells were selected with $2 \mu \mathrm{g} / \mathrm{ml}$ puromycin for 2 weeks to yield shRNAexpressing cells.

\section{siRNA transfection}

Small interfering RNAs (siRNAs) targeting HIF1 $\alpha$ (siHIF $1 \alpha-1, \quad$ GAGGAAGAACUAAAUCCAAdTdT; siHIF1 $\alpha-2$, UGAUACCAACAGUAACCAAdTdT), and negative control siRNA were purchased from GenePharma (Shanghai, China). The full-length MAP17 and its PDZ binding domain mutant MAP17-PDZm plasmids were synthesized by Shanghai Generay Biotech Co., Ltd. (Shanghai, China). HCC cells were transfected with siRNAs using Lipofectamine RNAiMax reagent (Invitrogen, USA) according to the manufacturer's protocol.

\section{Real-time quantitative PCR}

Total RNA was isolated from HCC cell lines using the RNAiso Plus reagent (Takara, Japan) and reversely transcribed through PrimeScript RT-PCR kit (Takara, Japan). The SYBR green-based real-time reverse transcriptionpolymerase chain reaction (RT-PCR) assay was used to determine gene expression on a 7500 Real-time PCR system (Applied Biosystems, Inc. USA). The housekeeping gene $\beta$-actin was served as an internal control for RNA quantity. Primers used in this study were shown as follows: MAP17 forward, 5'-TGGATGCAGGGCCTTATC G-3'; MAP17 reverse 5'-CGACGGTCAGGATCATGT GT-3'; HK2 forward, 5'-TTGACCAGGAGATTGACA TGGG-3'; HK2 reverse 5'-CAACCGCATCAGGACC TCA-3'; ENO1 forward, 5'-TGGTGTCTATCGAAGA TCCCTT-3'; ENO1 reverse 5'-CCTTGGCGATCCTC TTTGG-3'; LDHA forward, 5'-ATGGCAACTC TAAAGGATCAGC-3'; LDHA reverse 5'-CCAACC CCAACAACTGTAATCT- 3 '; $\beta$-actin forward, $5^{\prime}$-ACTC GTCATACTCCTGCT-3', $\beta$-actin reverse, $5^{\prime}$-GAAACT ACCTTCAACTCC-3'.

\section{Western blotting}

Whole-cell extracts were extracted using RIPA buffer $(150 \mathrm{mM} \mathrm{NaCl}, 50 \mathrm{mM}$ Tris- $\mathrm{HCl}$ at $\mathrm{pH} 7.4,1 \mathrm{mM}$ EDTA, $0.1 \%$ SDS, $1 \%$ Triton X-100, 1\% sodium deoxycholate and $1 \%$ NP-40) mixed with a protease and 
phosphatase inhibitor cocktail for $15 \mathrm{~min}$ on ice. The protein concentration was determined by a BCA Protein Assay Kit (Pierce Biotechnology, USA). Standard western blotting protocol was used and following antibodies were used in this study to analyze protein expression: MAP17 (1:1000, Abcam, ab156014), HIF1 $\alpha$ (1: 1000, Abcam, ab113642), p-P38 (1:1000, Cell Signaling Technology, \#4511), P38 (1:1000, Cell Signaling Technology, \#8690), p-S6K (1:1000, Cell Signaling Technology, \#9204), S6K (1:1000, Cell Signaling Technology, \#9202), p-Akt (1: 2000, Cell Signaling Technology, \#4060), Akt (1:1000, Cell Signaling Technology, \#4685), p-p44/42 MAPK (1: 1000, Cell Signaling Technology, \#4370), p44/42 MAPK (1:2000, Cell Signaling Technology, \#4695), p-STAT3 (1: 1000, Cell Signaling Technology, \#9145), STAT3 (1: 2000, Cell Signaling Technology, \#12640), PDZK1 (1: 1000, Santa Cruz Biotechnology, sc-390,964), and $\beta$ actin (1:2000, Abcam, ab8226).

\section{RNA sequencing analysis}

To illustrate the molecular mechanism of MAP17 in HCC, RNA sequencing was performed at sh-Ctrl and sh-MAP17 SMMC-7721 and HCC-LM3 cells. Preparation of the RNA library and sequencing were performed by Sinotech Genomics (Shenzhen, China). Gene expression was calculated using the Fragments Per Kilobase Million (FPKM) method. Differentially expressed genes were selected using a fold change expression cut-off of 2 and a $P$-value $<0.05$. Differentially expressed genes were further subjected to enrichment analysis by CytoScape software.

\section{Glucose uptake and lactate production}

HCC cells were seeded into a 6-well plate and cultured in a medium containing $4.5 \mathrm{~g} / \mathrm{L}$ glucose supplemented with $10 \%$ FBS under hypoxia or normoxia conditions. After $24 \mathrm{~h}$, the cell culture supernatants were collected and subjected for analysis. Glucose uptake was analyzed using the Amplex Red Glucose/Glucose Oxidase Assay Kit (A22189, Thermo Fisher Scientific, USA). Lactate production in the culture medium was determined by using the Lactate Assay Kit (K607-100, BioVision, USA). All results were normalized to the total protein amounts of cells that were measured by Pierce BCA Protein assay (Pierce Biotechnology, USA).

\section{Measurement of glycolytic capacity}

Extracellular acidification rate (ECAR) was analyzed by the Seahorse Bioscience XF96 Extracellular Flux Analyzer (Seahorse Bioscience, USA) with Seahorse XF Cell Glycolysis Stress Test Kit (Seahorse Bioscience, USA). Briefly, $2 \times 10^{4} /$ well HCC cells were seeded into an XF96-well plate and maintained overnight. The next day, cells were incubated with an unbuffered medium followed by sequential injection of $10 \mathrm{mM}$ glucose (Glc), $0.5 \mu \mathrm{M}$ oligomycin (Oligo), and $80 \mathrm{mM}$ 2-deoxyglucose (2-DG) to measure ECAR. Finally, the ECAR value was normalized to the protein amounts of cells before making comparisons between groups.

\section{Measurement of ROS levels}

For cellular ROS analysis, HCC cells were seeded in black 96-well plates at a density of $2 \times 10^{4}$ cells per well. Then cells were subjected to DCF-DA $(10 \mathrm{mmol} / \mathrm{L})$ staining in phenol red-free medium for $30 \mathrm{~min}$. Finally, the fluorescence intensity was measured immediately using a BioTek fluorescence plate reader.

\section{Measurement of HIF1a activity}

To determine the effect of MAP17 on HIF1 $\alpha$ transcriptional activity, nuclear extract lysates were obtained from MAP17 knockdown or MAP17-expressing HCC cells by using a Nuclear Extraction Kit (\#2900, Millipore). The activities of HIF $1 \alpha$ in nuclear extract lysates were detected using the HIF1 $\alpha$ Transcription Factor Assay Kit (ab133104, Abcam) according to the manufacturer's protocols.

\section{Proximity ligation assay}

Proximity ligation assay (PLA, Olink Bioscience, DUO92007) was performed as reported previously [24]. Briefly, SMMC-7721 and HCC-LM3 cells seeded onto IBIDI $\mu$-Dish and fixed with $4 \%$ paraformaldehyde, followed by permeabilization using $0.1 \%$ Triton- $\mathrm{X}$ and blocked with 5\% BSA in PBS. Mouse anti-PDZK1 (1:200, Santa Cruz Biotechnology, sc-390,964) and rabbit antiMAP17 (1:100, Abcam, ab156014) were used as primary antibodies. The next day, cells were incubated with the anti-mouse and anti-rabbit probes. Ligation and amplification reactions were carried out according to the manufacturer's protocols. Cells were then washed, and the nuclei were stained with DAPI. Finally, the nuclei and proximity ligated foci were visualized on a confocal microscope.

\section{Co-immunoprecipitation experiment}

The supernatants were pre-incubated with protein A/G PLUS agarose (Santa Cruz Biotechnology, sc-2003), and subsequently incubated with $2 \mu \mathrm{g}$ of MAP17 antibodies or control IgG overnight at $4{ }^{\circ} \mathrm{C}$, followed by addition of $20 \mu \mathrm{l}$ protein $\mathrm{A} / \mathrm{G}$ agarose for $2 \mathrm{~h}$ at room temperature. After washing three times with lysis buffer, immunoprecipitates were boiled in $1 \times$ loading buffer and subjected for standard western blotting analysis.

\section{Chromatin immunoprecipitation-PCR}

Chromatin Immunoprecipitation (ChIP) experiment was performed in SK-H929, MHCC-97H, and Huh7 cells 
under $20 \% \mathrm{O}_{2}$ and $1 \% \mathrm{O}_{2}$ condition. In brief, HCC cells were fixed with $1 \%$ formaldehyde for $10 \mathrm{~min}$ at room temperature followed by incubation with $0.125 \mathrm{M}$ glycine for $10 \mathrm{~min}$. Cells were washed with ice-cold PBS for three times. Then, fixed cells were collected and resuspended in lysis buffer, supplemented with protease inhibitor cocktails, and sonicated to obtain chromatin fragments of about 500-1500 bp. The supernatants were collected and incubated overnight with anti-HIF1 $\alpha$ antibody (F7425) and Dynabeads Protein G. The beads were washed, and the precipitated chromatin complexes were collected, purified, and de-crosslinked. Finally, the precipitated DNA fragments were analyzed by RT-PCR.

\section{Plate colony formation assay}

HCC cells were counted and seeded in 6-well plates with 500-1000 cells per well. Cells were allowed to grow for 1014 days and the culture medium was replaced every three days. Finally, the cells were fixed with $4 \%$ paraformaldehyde, and colonies were counted after staining with $0.1 \%$ crystal violet for $20 \mathrm{~min}$. All the experiments were performed in triplicate and repeated three times independently.

\section{In vivo tumorigenicity}

To generate subcutaneous xenograft, $2 \times 10^{6}$ SMMC7721 and HCC-LM3 cells stably transfected with shMAP17 or sh-Ctrl were suspended in $100 \mu \mathrm{L}$ PBS and then inoculated subcutaneously into the BALB/c nude mice (male, 6-week old). The volume of xenograft neoplasms was monitored and estimated as follows: tumor volume $=$ length $\times$ width $^{2} / 2$. At the endpoint of the experiment, the mice were sacrificed and the xenograft neoplasms were harvested and weighed. This study was approved by the Research Ethics Committee of Shanghai Jiao Tong University carried out following the guidelines of the national animal protection and ethics institute.

\section{Statistical analysis}

All the data were presented as means \pm SEM. Differences were compared using two-tailed Student's t-test or one-way ANOVA followed by post hoc Duncan tests. The GraphPad Prism (GraphPad Software Inc., San Diego, CA) was used for statistical analyses. The prognostic analysis was conducted by the Kaplan-Meier method and analyzed by the log-rank test. A $P$ value of less than 0.05 was considered statistically significant.

\section{Results}

\section{Hypoxia-dependent overexpression of MAP17 in HCC}

To determine the critical regulators involved in the hypoxic tumor microenvironment and tumor glycolysis, we performed an integrated analysis by leveraging molecular profiles from TCGA cohort. Based on the hypoxiarelated gene signature reported previously [25], we used a dichotomy method (hypoxia-high vs. hypoxia-low) and identified 742 differentially expressed mRNAs associated with hypoxia. Likewise, 285 glycolysis-related genes were also found in HCC. Among these genes, 4 genes (LOXL2, MAP17, LDHA, and EDIL3) were highly expressed in HCC (Fig. 1a). Given the roles and mechanisms of LOXL2, LDHA, and EDIL3 have been well documented in HCC [26-28], MAP17 is selected for further investigation. MAP17 is almost not expressed in normal human tissues except for the kidney (Supplementary Figure 1). To confirm the expression pattern of MAP17 in $\mathrm{HCC}$, we analyzed its expression in a tissue microarray containing 202 pathologist-certified HCC samples by immunohistochemical method. As shown in Fig. 1b, MAP17 immunoreactivity was largely present at the tumor cell membrane and merely distributed in nontumor tissues. Kaplan-Meier curve revealed that HCC patients with higher MAP17 levels had a significantly reduced overall survival (Fig. 1c). By real-time qPCR and western blotting analysis, we noticed that SMMC-7721 and HCC-LM3 cells had a higher endogenous level of MAP17 (Fig. 1d). To certify whether MAP17 is induced by hypoxia in HCC, we cultured three HCC cell lines (SK-H929, MHCC-97H, and Huh7) under both normoxic and hypoxic conditions. As a result, MAP17 expression was significantly boosted by hypoxia at both the mRNA (Fig. 1e) and protein levels (Fig. If and Supplementary Figure 2), indicating a regulatory role of HIF1 $\alpha$ in MAP17 expression. As the next line of evidence, genetic silencing of HIF1 $\alpha$ largely attenuated hypoxiainduced MAP17 expression (Fig. 1g-h). Finally, chromatin immunoprecipitation data showed that HIF1 $\alpha$ interacted directly with MAP17 gene promoters under hypoxia (Fig. 1i). Therefore, highly expressed MAP17 may be induced by hypoxia in HCC. In addition to HCC, MAP17 was also overexpressed in many other types of tumors, such as cervical cancer, colorectal cancer, lung cancer, ovarian cancer, pancreatic cancer, gastric cancer, thyroid cancer, uterine corpus endometrial carcinoma, and uterine carcinosarcoma (Supplementary Figure 3), suggesting that dysregulation of MAP17 is a universal phenomenon in human cancers.

\section{MAP17 promotes the Warburg effect in HCC cells}

To demonstrate whether MAP17 plays a role in HCC glycolysis, in vitro loss-of-function studies were first carried out. Five independent shRNAs against MAP17 were used and two shRNAs (sh-MAP17-\#3 and sh-MAP17-\#5) led to a $>60 \%$ reduction of MAP17 protein expression in both SMMC-7721 and HCC-LM3 cells (Fig. 2a). Consistent with the knockdown efficiency, reduction in MAP17 expression led to a significant decrease in glucose uptake (Fig. 2b), lactate production (Fig. 2c), extracellular acidification rate (Fig. 2d), and expression of glycolytic genes 


\section{A}

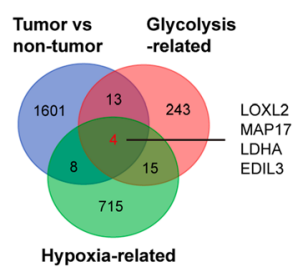

C

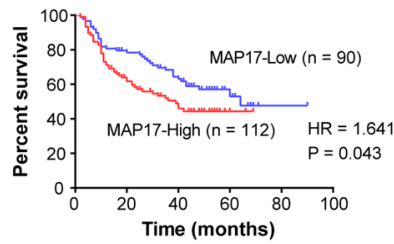

E

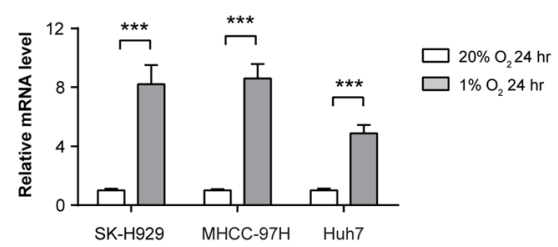

G

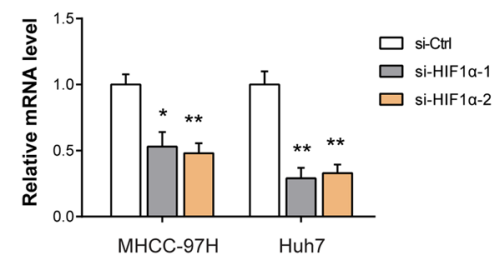

I

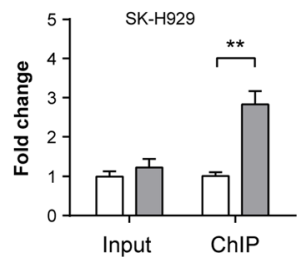

B

D
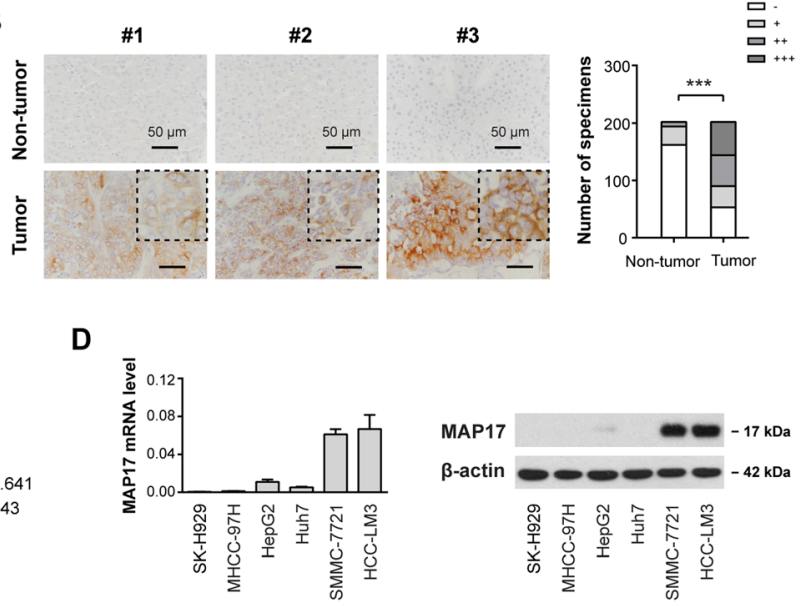

$\mathbf{F}$

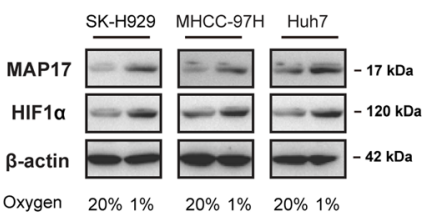

H

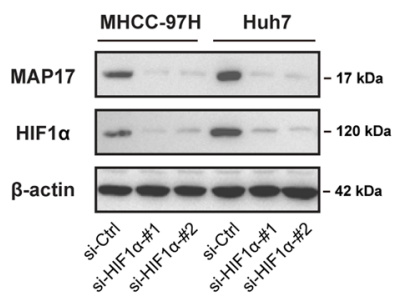

몬.

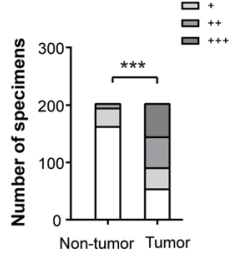$$
\text { . }
$$ 


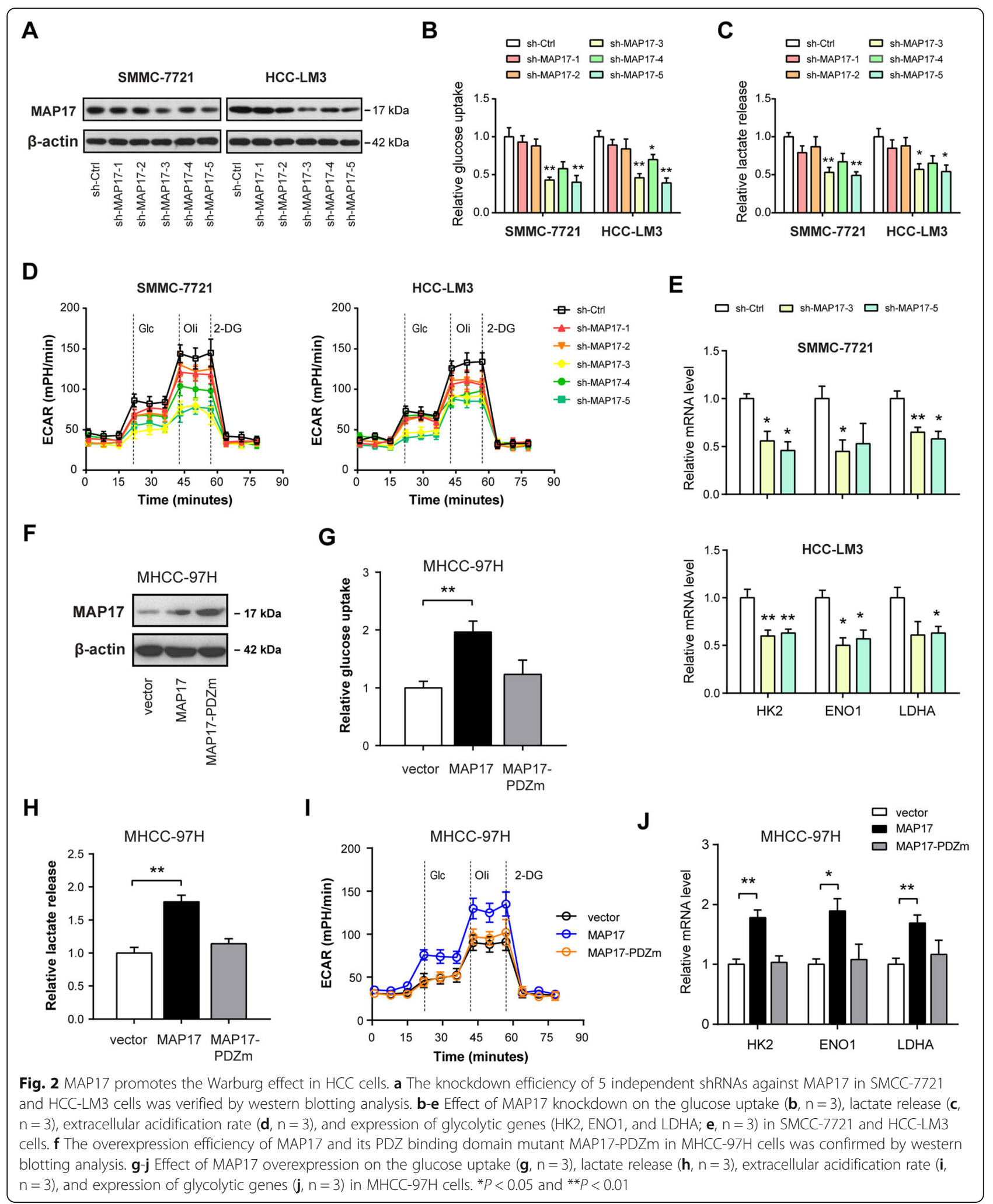

MAP17 is required for tumor growth in HCC

Next, we studied the potential oncogenic roles of MAP17 in HCC. By plate colony formation assay, we found that MAP17 knockdown contributed to decreased proliferative capabilities in SMMC-7721 and HCC-LM3 cells (Fig. 3a). In the subcutaneous xenograft model, 
MAP17 knockdown remarkably retarded tumor growth as demonstrated by tumor volume, tumor weight, and the expression of proliferation index Ki67 (Fig. 3b and Supplementary Figure 4). In contrast, MAP17 but not MAP17-PDZm overexpression enhanced the colony formation ability of MHCC-97H cells (Fig. 3c), suggesting that MAP17-mediated increase in tumorigenesis is also dependent on its PDZ-binding domain. To determine whether MAP17-dependent tumorigenesis is coupled to glycolysis, we replaced glucose in the culture medium with galactose, which occurs at a much lower rate than glucose entry into glycolysis and thereby suppressing glycolytic flux. As shown in Fig. 3d, galactose largely compromised the growth-promoting role induced by MAP17. Collectively, these findings above indicate that MAP17 may coordinate the Warburg effect to promote tumor growth in HCC.

\section{MAP17 regulates intracellular ROS to enhance the tumorigenic and glycolytic phenotypes}

It is well known that MAP17 interacts with PDZK1 and other members of the NHeRF (sodium hydrogen exchange regulatory factor) family $[15,29]$. Through proximity ligation assay and co-immunoprecipitation assay, we observed a physical interaction between MAP17 and PDZK1 in SMMC-7721 and HCC-LM3 cells (Supplementary Figure 5A-B). Interaction between MAP17 and PDZK1 potentiates SGLT1/2-mediated glucose uptake in a sodium-dependent manner in Xenopus oocytes and mammary cells $[16,30]$. However, since SGLT1/2 expression was not present in HCC tissues (Supplementary Figure $5 \mathrm{C}$ ), we ruled out the possibility of its role in MAP17-induced glycolysis. ROS is a key mediator in many signaling cascades that are related to cell proliferation and transformation [31]. Although MAP17 has no enzymatic activity, its overexpression can lead to an increase of $30-40 \%$ in ROS generation [32]. Indeed, we found that MAP17 knockdown resulted in a $~ 40 \%$ reduction of ROS in SMMC-7721 and HCC-LM3 cells (Fig. 4a), while MAP17 overexpression increased ROS level in MHCC-97H cells (Fig. 4b). To test whether ROS is responsible for the enhanced tumorigenic properties of MAP17 in HCC, we blocked ROS with the antioxidant $\mathrm{N}$-acetylcysteine (NAC). In MHCC-97H cells overexpressing MAP17, NAC treatment significantly reduced the glycolytic phenotypes of MAP17 (Fig. 4c-e). In addition, the number of colonies of MAP17-expressing cells was also decreased by NAC (Fig. 4f).

\section{AKT and HIF1a are the downstream effectors of ROS in $\mathrm{HCC}$}

To pursue the cellular mechanism of MAP17, we performed RNA sequencing analysis in sh-Ctrl and shMAP17 HCC-LM3 cells. The result showed that a total of 1909 genes whose expressions were downregulated in response to MAP17 knockdown in HCC-LM3 cells. Interestingly, functional enrichment analysis of these genes demonstrated significant enrichment of ROS metabolic process, cell surface receptor signaling, cell communication, mitotic cell cycle progression, and regulation of cell differentiation (Fig. 5a). ROS act as a second messenger can activate many signaling pathways, such as $\mathrm{p} 42 / \mathrm{p} 44$ mitogen-activated protein kinase (MAPK), p38 MAPK, signal transducers and activators of transcription (STAT), Akt/protein kinase B, p70S6K, and HIF1 $\alpha$ [32]. By western blotting analysis, we found that MAP17 knockdown significantly inhibited the activation of AKT and HIF1 $\alpha$ but not p42/p44, p38, STAT3, and p70S6K in SMMC-7721 and HCC-LM3 cells (Fig. 5b). Conversely, MAP17 overexpression enhanced AKT activation, which was downregulated by the addition of $10 \mathrm{mM}$ NAC (Fig. 5c). The real-time qPCR results showed that genetic manipulation of MAP17 failed to affect HIF $1 \alpha$ mRNA expression in HCC cells (Supplementary Figure 6). However, HIF1 $\alpha$ protein level (Fig. 5d) and transcriptional activity (Fig. 5e) were significantly enhanced by MAP17 overexpression and further blocked by neutralization of ROS with NAC, suggesting that MAP17 may exert a posttranslational regulatory role on HIF1 $\alpha$ protein stability. Indeed, overexpression of MAP17 reduced the degradation rate of HIF1 $\alpha$ protein in MHCC-97H cells (Fig. 5f). Moreover, cells used for MAP17 knockdown or overexpression were pretreated with MG132 to prevent HIF1 $\alpha$ from being degraded under hypoxic conditions. As a result, significantly less HIF1 $\alpha$ was accumulated during MG132 treatment following MAP17 knockdown; in contrast, more HIF1 $\alpha$ was accumulated following MAP17 overexpression (Fig. 5g). To confirm whether AKT and HIF1 $\alpha$ mediate the roles of MAP17 in HCC, a specific inhibitor of AKT (MK-2206) and specific siRNAs against HIF1 $\alpha$ were used in MHCC-97H cells. Expectedly, MAP17dependent glycolysis and tumor growth were effectively blocked by MK-2206 (Supplementary Figure 7) or HIF1 $\alpha$ knockdown (Supplementary Figure 8). Taken together, AKT and HIF1 $\alpha$ may act as the downstream effectors of the MAP17/ROS axis in HCC.

\section{MAP17 correlates p-AKT and HIF1a in clinical HCC samples}

Finally, we determined the expression of p-AKT and HIF1 $\alpha$ by IHC in a cohort of 202 HCC patients. As a result, there was a significantly positive correlation between MAP17 expression and $\mathrm{p}$-AKT (Pearson $\mathrm{R}=$ 0.382; $P<0.001$ ) or HIF1 $\alpha$ expression (Pearson $R=$ 0.433; $\mathrm{P}<0.001$ ) in the HCC samples (Fig. 6a). Moreover, we tested TCGA cohort and found that MAP17 expression was closely associated with hypoxia gene 


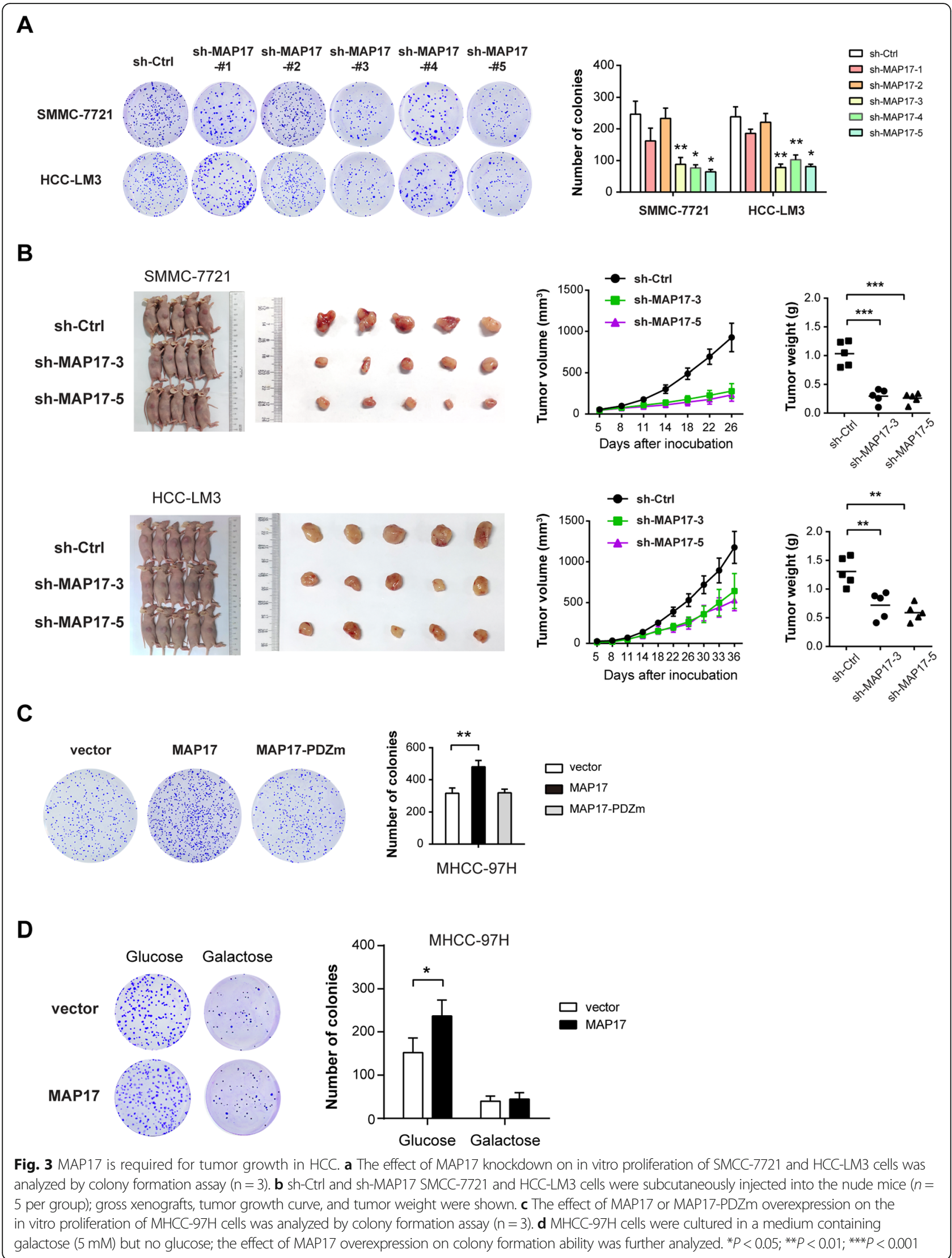




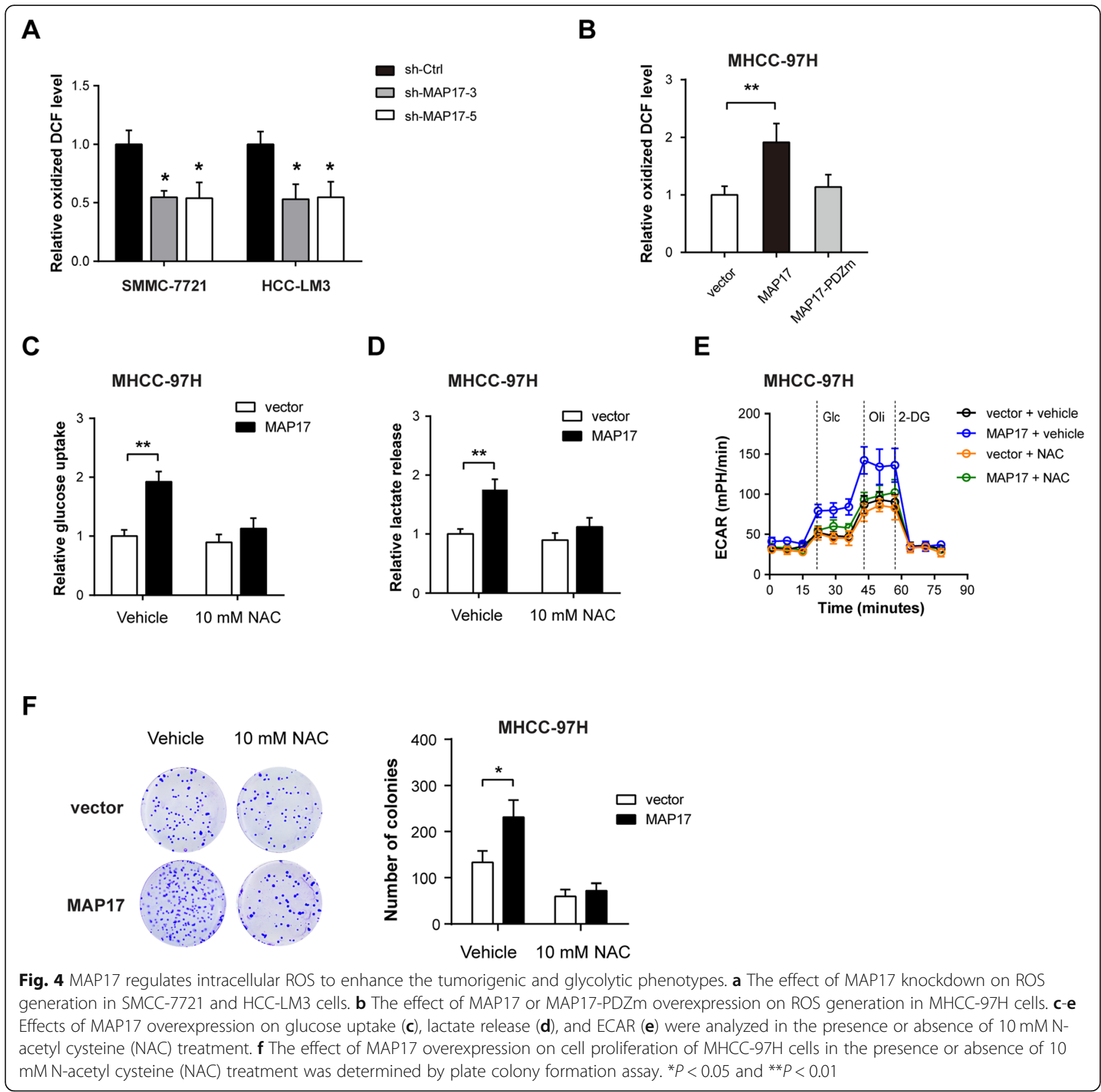

signature and PI3K/AKT pathway (Fig. 6b). These findings further confirm the molecular mechanism of MAP17 in the clinical setting.

\section{Discussion}

Metabolic reprogramming in the tumor microenvironment is commonly seen in a wide spectrum of human solid cancers and emerged as a central contributor to the tumor progression machinery. Dysregulated growth factor signaling, activation of HIF- $1 \alpha$-transcription, activation of oncogenes or loss-of-function of suppressor genes contributes to cancer metabolic reprogramming. In the present study, our integrated analysis for the first time identified MAP17 as a major glycolytic regulator in response to hypoxic tumor microenvironment. Through regulation of AKT activation and HIF1 $\alpha$, MAP17 facilitates the Warburg effect to promote tumor growth in HCC (Fig. 6c).

In normal human tissues, MAP17 is dominantly restricted to epithelial cells from the kidney. However, MAP17 overexpression has been reported in many tumor types, such as lung cancer, breast cancer, laryngeal cancer, rectal cancer, and prostate cancer [19, 3335]. Notably, increased MAP17 expression was present at advanced or metastatic tumors and associated with tumor progression. In HCC, MAP17 expression is 
A

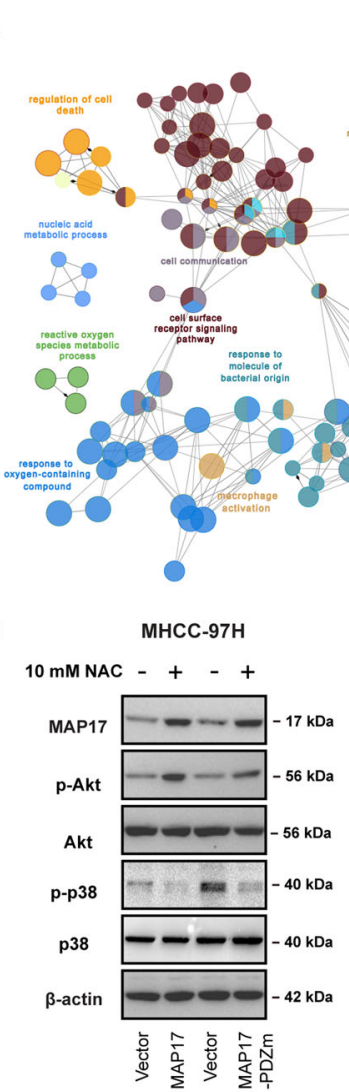

F

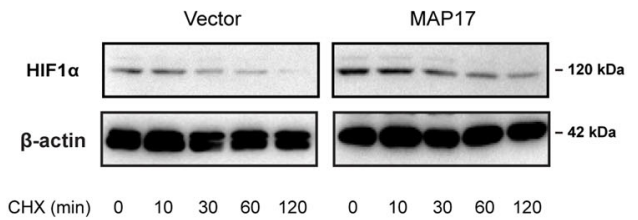

B

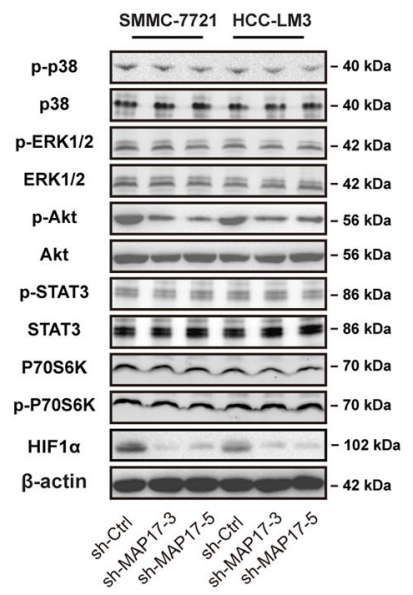

E

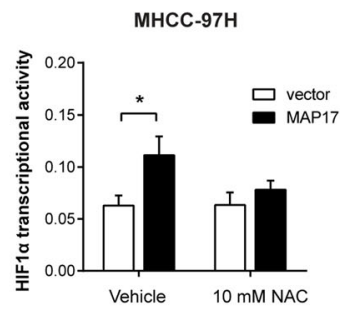

\section{.}

G

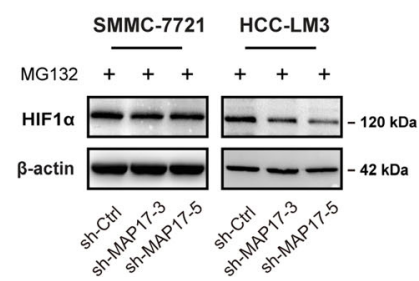

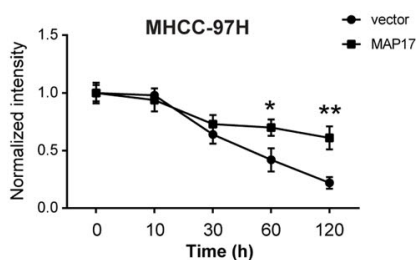

$10 \mathrm{mMNAC}-++2+$

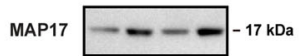

HIF1 $\alpha--120 \mathrm{kDa}$

$\beta$-actin $=--42 \mathrm{kDa}$

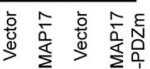




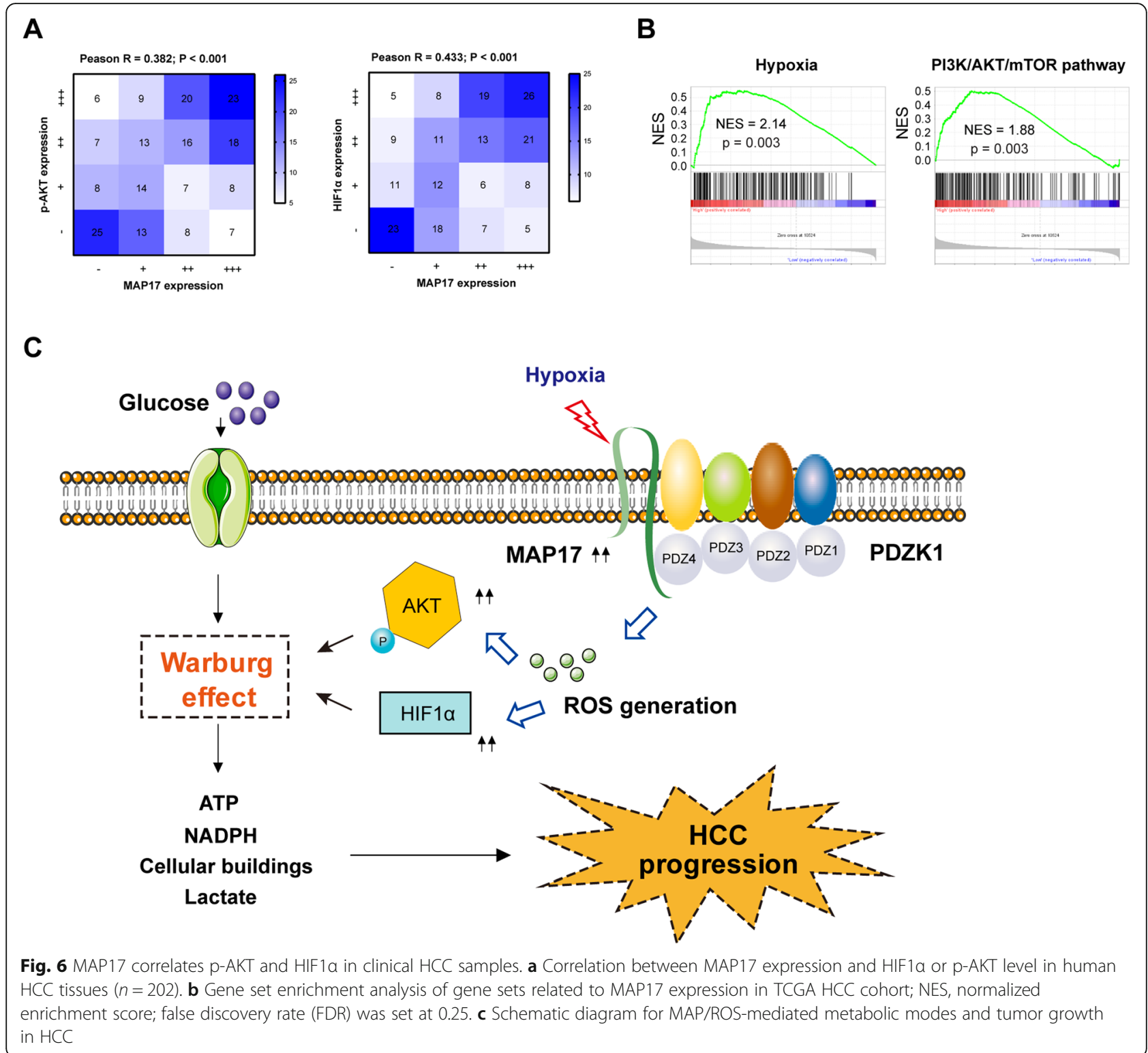

positively correlated with gender, distant metastasis, early recurrence, and serum alpha-fetoprotein [36]. Consistent with this finding, we confirmed that higher MAP17 expression is associated with a poor prognosis, indicating that MAP17 may be a novel prognostic biomarker for HCC patients. In normal human epidermal keratinocytes, MAP17 is upregulated in response to interferon-gamma, interleukin 4 (IL-4), IL-6, IL-17A, or IL-22 [37]. However, little is known about the reason for dysregulated MAP17 in cancers. Here, our findings explained that hypoxia acts as an inducer for MAP17 expression in HCC. MAP17 plays pleiotropic oncogenic roles in several human cancers, such as cell apoptosis [38], cell invasion [36], tumor growth [20, 39], chemotherapy sensitivity $[35,40,41]$, epithelial mesenchymal transition (EMT) [42], stem cell-like properties [17], and inflammatory response $[14,18]$. In this study, we presented a novel regulatory role of MAP17 in the Warburg effect, which further broadens the current knowledge of the hypoxia-induced changes in metabolism during HCC development. Notably, increasing evidence has shown that metabolic reprogramming other than Warburg effect is also essential for tumor development and progression. Indeed, other metabolic features, in particular, the reverse Warburg effect, metabolic symbiosis, and addiction to glutamine metabolism, create challenges for anti-cancer treatment due to adaptive or acquired chemoresistance. Therefore, a comprehensive understanding of metabolic reprogramming may facilitate the development of promising therapeutic strategies by 
repositioning drug (such as metformin) targeting metabolic reprogramming [43].

The C-terminal sequence of MAP17 contains a PDZbinding domain, which allows its interaction with PDZ domain-containing proteins, especially PDZK1. Specifically, the MAP17 PDZ-binding domain is essential for its oncogenic activities. For instance, disruption of this sequence of MAP17 by point mutations abolishes its ability to promote the tumorigenic capability of malignant melanoma cells [20]. In line with this observation, we found that ectopic expression of PDZ binding domain mutant MAP17-PDZm cannot promote the Warburg effect or play a growth-promoting effect in HCC. The interaction of MAP17 with SGLT1/2 proteins can increase glucose uptake to enhance glycolytic flux $[16,30]$. Interestingly, we revealed that MAP17-induced glycolysis is not dependent on SGLT1/2 in HCC. MAP17 has been reported to activate AKT/mTOR pathway in $\mathrm{HCC}$ cells [36]. However, the detailed molecular mechanism remains unclear. Accumulated evidence suggests that the tumorigenic roles of MAP17 are closely associated with an increase in ROS generation. Indeed, our results supported that ROS is generated in a MAP17-dependent manner and is responsible for MAP17-mediated glycolytic and oncogenic activities. Indeed, ROS removal by treatment of antioxidant NAC largely compromised the tumorigenic roles of MAP17. ROS might act as an intracellular signal or a second messenger to initiate downstream cascades that induce malignant phenotypes in cancers. In this study, we revealed that MAP17 triggers a ROS-dependent AKT and HIF $1 \alpha$ activation. AKT can modulate aerobic glycolysis through multiple pathways, including but not limited to activation of HIF $1 \alpha$, mTOR, $\mathrm{c}-\mathrm{Myc}$, and the subsequent expression of glycolytic enzymes [44]. HIF1 $\alpha$ is a well-known transcriptional factor for the Warburg effect [45]. Thus, it is reasonable that AKT and HIF1 $\alpha$ are functional mediators of MAP17 in HCC. Although we uncovered a mechanism of MAP17dependent glycolysis in HCC, the reason for how ROS is increased by MAP17 is not known and warrants further investigations. One possibility is that a direct interaction with the membrane transporters, changing the intracellular redox level through altering the intra/extracellular ion balance [32]. In addition, MAP17 can activate the Notch pathway to promote stem cell-like properties through direct interaction with NUMB via the PDZbinding domain [17]. Therefore, we cannot rule out other possibilities that are responsible for MAP17dependent glycolysis in HCC.

\section{Conclusions}

We report here that the hypoxic tumor microenvironment may induce MAP17 expression in HCC. Moreover, we elucidate that MAP17-induced ROS activate AKT and increase HIF1 $\alpha$ protein stability to promote the Warburg effect and tumor growth. Therefore, MAP17 could function as a glycolysis enhancer in $\mathrm{HCC}$, and targeting the MAP17/ROS axis may represent a new method for the treatment of HCC.

\begin{abstract}
Abbreviations
HCC: Hepatocellular carcinoma; ROS: Reactive oxygen species; HIF1a: Hypoxia-inducible factor 1a; SGLT1/2: Sodium-dependent glucose transporter 1/2; RT-PCR: Reverse transcription-polymerase chain reaction; FPKM: Fragments Per Kilobase Million; ECAR: Extracellular acidification rate; 2DG: 2-deoxyglucose; NAC: N-acetylcysteine
\end{abstract}

\section{Supplementary Information}

The online version contains supplementary material available at https://doi. org/10.1186/s13046-021-01927-5

Additional file 1: Supplementary Figure 1. Expression pattern of MAP17 in normal human tissues and cells. Supplementary Figure 2. Expression of MAP17 under hypoxic condition in HCC cells. Supplementary Figure 3. Expression pattern of MAP17 in human cancers. Supplementary Figure 4. MAP17 knockdown inhibits tumor growth in vivo. Supplementary Figure 5. Interaction between MAP17 and PDZK1 in HCC cells. Supplementary Figure 6. The effect of MAP17 knockdown or overexpression on HIF1a mRNA expression in HCC. Supplementary Figure 7. MK-2206 blocks the tumorigenic and glycolytic phenotypes induced by MAP17. Supplementary Figure 8. HIF1a knockdown blocks the tumorigenic and glycolytic phenotypes induced by MAP17.

\section{Acknowledgements}

The research was supported by grants from National Key R\&D Program of China (2018YFC2002000), National Natural Science Foundation of China (82001469, 81701374 and 82071581), Young Excellent Medical Talents Training Program of Health Department of Shanghai Municipal Government (2018YQ58), and Shanghai Sailing Program (20YF1412000).

\section{Authors' contributions}

Zhijun Bao and Mingxuan Feng conceived the study plan and contributed to the revision of the final manuscript. Fangyuan Dong, Rongkun Li, ShuHeng Jiang, Xiaona Hu, Yan Zhang, and Jianfeng Yao performed the experiments, analyzed the data and finished the manuscript writing. Jiaofeng Wang contributed to the in vitro experiments. Fangyuan Dong and Rongkun Li performed analysis and interpretation of data. All authors contributed to the writing and reviewing of the manuscript, and approved the final manuscript for submission.

\section{Funding}

The research was supported by grants from National Key R\&D Program of China (2018YFC2002000), National Natural Science Foundation of China (82001469, 81701374 and 82071581), Young Excellent Medical Talents Training Program of Health Department of Shanghai Municipal Government (2018YQ58), and Shanghai Sailing Program (20YF1412000).

\section{Availability of data and materials}

All data generated or analyzed during this research are included in this manuscript.

\section{Declarations}

\section{Ethics approval and consent}

All human samples were obtained with informed consent. All animals received humane care according to the criteria outlined in the "Guide for the Care and Use of Laboratory Animals" prepared by the National Academy of Sciences and published by the National Institutes of Health. 


\section{Consent for publication}

We have obtained consent to publish from the participant to report individual patient data.

\section{Competing interests}

The authors declare that they have no competing interests.

\begin{abstract}
Author details
'Department of Gastroenterology, Huadong Hospital, Shanghai Medical College, Fudan University, No.221 Yan'an West Road, Shanghai 200040, P.R. China. ${ }^{2}$ Shanghai Key Laboratory of Clinical Geriatric Medicine, Shanghai 200040, P.R. China. ${ }^{3}$ Research Center on Aging and Medicine, Fudan University, Shanghai 200040, P.R. China. ${ }^{4}$ Department of Geriatrics, Huadong Hospital, Shanghai Medical College, Fudan University, Shanghai 200040, P.R. China. ${ }^{5}$ Institute of Oncology, Affiliated Hospital of Jiangsu University, Zhenjiang 212001, China. ' ${ }^{6}$ State Key Laboratory of Oncogenes and Related Genes, Shanghai Cancer Institute, Ren Ji Hospital, School of Medicine, Shanghai Jiao Tong University, Shanghai 200240, P.R. China. 'Department of Liver Surgery, Ren Ji Hospital, School of Medicine, Shanghai Jiao Tong University, 1630 Dongfang Road, Shanghai 200127, P.R. China.
\end{abstract}

Received: 30 November 2020 Accepted: 24 March 2021

Published online: 08 April 2021

\section{References}

1. Forner A, Reig M, Bruix J. Hepatocellular carcinoma. Lancet. 2018;391(10127): 1301-14. https://doi.org/10.1016/S0140-6736(18)30010-2.

2. Abou-Alfa GK, Meyer T, Cheng AL, El-Khoueiry AB, Rimassa L, Ryoo BY, et al. Cabozantinib in patients with advanced and progressing hepatocellular carcinoma. N Engl J Med. 2018;379(1):54-63. https://doi.org/10.1056/ NEJMoa1717002.

3. Villanueva A. Hepatocellular Carcinoma. N Engl J Med. 2019;380(15):1450-62. https://doi.org/10.1056/NEJMra1713263.

4. Hanahan D, Weinberg RA. Hallmarks of cancer: the next generation. Cell. 2011;144(5):646-74. https://doi.org/10.1016/j.cell.2011.02.013.

5. Vander Heiden MG, Cantley LC, Thompson CB. Understanding the Warburg effect: the metabolic requirements of cell proliferation. Science. 2009; 324(5930):1029-33. https://doi.org/10.1126/science.1160809.

6. Feng J, Li J, Wu L, Yu Q, Ji J, Wu J, et al. Emerging roles and the regulation of aerobic glycolysis in hepatocellular carcinoma. J Exp Clin Cancer Res. 2020;39(1):126. https://doi.org/10.1186/s13046-020-01629-4.

7. lansante V, Choy PM, Fung SW, Liu Y, Chai JG, Dyson J, et al. PARP14 promotes the Warburg effect in hepatocellular carcinoma by inhibiting JNK1-dependent PKM2 phosphorylation and activation. Nat Commun. 2015; 6(1):7882. https://doi.org/10.1038/ncomms8882.

8. Lin YH, Wu MH, Huang YH, Yeh CT, Cheng ML, Chi HC, et al. Taurine upregulated gene 1 functions as a master regulator to coordinate glycolysis and metastasis in hepatocellular carcinoma. Hepatology. 2018;67(1):188-203. https://doi.org/10.1002/hep.29462.

9. Keith B, Simon MC. Hypoxia-inducible factors, stem cells, and cancer. Cell. 2007;129(3):465-72. https://doi.org/10.1016/j.cell.2007.04.019.

10. Cairns RA, Harris IS, Mak TW. Regulation of cancer cell metabolism. Nat Rev Cancer. 2011;11(2):85-95. https://doi.org/10.1038/nrc2981.

11. Dang CV. MYC on the path to cancer. Cell. 2012;149(1):22-35. https://doi. org/10.1016/j.cell.2012.03.003.

12. Sukonina V, Ma H, Zhang W, Bartesaghi S, Subhash S, Heglind M, et al. FOXK1 and FOXK2 regulate aerobic glycolysis. Nature. 2019;566(7743):27983. https://doi.org/10.1038/s41586-019-0900-5.

13. Li L, Liang Y, Kang L, Liu Y, Gao S, Chen S, et al. Transcriptional regulation of the Warburg effect in Cancer by SIX1. Cancer Cell. 2018;33(3):368-85 e367. https://doi.org/10.1016/j.ccell.2018.01.010.

14. Garcia-Heredia JM, Carnero A, Dr. Jekyll and Mr. Hyde. MAP17's upregulation, a crosspoint in cancer and inflammatory diseases. Mol Cancer. 2018;17:80.

15. Lanaspa MA, Giral H, Breusegem SY, Halaihel N, Baile G, Catalan J, et al. Interaction of MAP17 with NHERF3/4 induces translocation of the renal Na/ pi lla transporter to the trans-Golgi. Am J Physiol Renal Physiol. 2007;292(1): F230-42. https://doi.org/10.1152/ajprenal.00075.2006.

16. Blasco T, Aramayona JJ, Alcalde Al, Catalan J, Sarasa M, Sorribas V. Rat kidney MAP17 induces cotransport of Na-mannose and Na-glucose in
Xenopus laevis oocytes. Am J Physiol Renal Physiol. 2003;285(4):F799-810. https://doi.org/10.1152/ajprenal.00149.2003.

17. Garcia-Heredia JM, Lucena-Cacace A, Verdugo-Sivianes EM, Perez M, Carnero A. The cargo protein MAP17 (PDZK1IP1) regulates the Cancer stem cell Pool activating the notch pathway by abducting NUMB. Clin Cancer Res. 2017; 23(14):3871-83. https://doi.org/10.1158/1078-0432.CCR-16-2358.

18. Garcia-Heredia JM, Carnero A. The cargo protein MAP17 (PDZK1IP1) regulates the immune microenvironment. Oncotarget. 2017;8(58):98580-97. https://doi.org/10.18632/oncotarget.21651.

19. Rivero M, Peinado-Serrano J, Munoz-Galvan S, Espinosa-Sanchez A, SuarezMartinez E, Felipe-Abrio B, et al. MAP17 (PDZK1IP1) and pH2AX are potentia predictive biomarkers for rectal cancer treatment efficacy. Oncotarget. 2018; 9(68):32958-71. https://doi.org/10.18632/oncotarget.26010.

20. Guijarro MV, Leal JF, Blanco-Aparicio C, Alonso S, Fominaya J, Lleonart M, et al. MAP17 enhances the malignant behavior of tumor cells through ROS increase. Carcinogenesis. 2007;28:2096-104.

21. Guijarro MV, Leal JF, Fominaya J, Blanco-Aparicio C, Alonso S, Lleonart M, et al. MAP17 overexpression is a common characteristic of carcinomas. Carcinogenesis. 2007;28(8):1646-52. https://doi.org/10.1093/carcin/bgm083.

22. Tang Z, Li C, Kang B, Gao G, Li C, Zhang Z. GEPIA: a web server for cancer and normal gene expression profiling and interactive analyses. Nucleic Acids Res. 2017;45(W1):W98-W102. https://doi.org/10.1093/nar/gkx247.

23. Dong F, Yang Q, Wu Z, Hu X, Shi D, Feng M, et al. Identification of survivalrelated predictors in hepatocellular carcinoma through integrated genomic, transcriptomic, and proteomic analyses. Biomed Pharmacother. 2019;114: 108856. https://doi.org/10.1016/j.biopha.2019.108856.

24. Jiang SH, Li J, Dong FY, Yang JY, Liu DJ, Yang XM, et al. Increased serotonin signaling contributes to the Warburg effect in pancreatic tumor cells under metabolic stress and promotes growth of pancreatic tumors in mice. Gastroenterology. 2017;153(1):277-91 e219. https://doi.org/10.1053/j.gastro.2 017.03.008.

25. Ye Y, Hu Q, Chen H, Liang K, Yuan Y, Xiang Y, et al. Characterization of hypoxia-associated molecular features to aid hypoxia-targeted therapy. Nat Metab. 2019;1(4):431-44. https://doi.org/10.1038/s42255-019-0045-8.

26. Wong CC, Tse AP, Huang YP, Zhu YT, Chiu DK, Lai RK, et al. Lysyl oxidaselike 2 is critical to tumor microenvironment and metastatic niche formation in hepatocellular carcinoma. Hepatology. 2014;60(5):1645-58. https://doi. org/10.1002/hep.27320.

27. Sheng SL, Liu JJ, Dai YH, Sun XG, Xiong XP, Huang G. Knockdown of lactate dehydrogenase a suppresses tumor growth and metastasis of human hepatocellular carcinoma. FEBS J. 2012;279(20):3898-910. https://doi.org/1 0.1111/j.1742-4658.2012.08748.X.

28. Feng MX, Ma MZ, Fu Y, Li J, Wang T, Xue F, et al. Elevated autocrine EDIL3 protects hepatocellular carcinoma from anoikis through RGD-mediated integrin activation. Mol Cancer. 2014;13(1):226. https://doi.org/10.1186/14 76-4598-13-226.

29. Pribanic S, Gisler SM, Bacic D, Madjdpour C, Hernando N, Sorribas V, et al. Interactions of MAP17 with the NaPi-lla/PDZK1 protein complex in renal proximal tubular cells. Am J Physiol Renal Physiol. 2003;285(4):F784-91. https://doi.org/10.1152/ajprenal.00109.2003.

30. Coady MJ, El Tarazi A, Santer R, Bissonnette P, Sasseville LJ, Calado J, et al. MAP17 is a necessary activator of renal Na+/glucose Cotransporter SGLT2. J Am Soc Nephrol. 2017:28(1):85-93. https://doi.org/10.1681/ASN.2015111282.

31. Moloney JN, Cotter TG. ROS signalling in the biology of cancer. Semin Cell Dev Biol. 2018:80:50-64. https://doi.org/10.1016/.jsemcdb.2017.05.023.

32. Carnero A. MAP17 and the double-edged sword of ROS. Biochim Biophys Acta. 2012;1826(1):44-52. https://doi.org/10.1016/j.bbcan.2012.03.004.

33. Tampaki EC, Tampakis A, Nonni A, von Flue M, Patsouris E, Kontzoglou K, et al. Combined Fascin-1 and MAP17 expression in breast Cancer identifies patients with high risk for disease recurrence. Mol Diagn Ther. 2019;23(5): 635-44. https://doi.org/10.1007/s40291-019-00411-3.

34. Perez M, Praena-Fernandez JM, Felipe-Abrio B, Lopez-Garcia MA, LucenaCacace A, Garcia A, et al. MAP17 and SGLT1 protein expression levels as prognostic markers for cervical tumor patient survival. PLoS One. 2013;8(2): e56169. https://doi.org/10.1371/journal.pone.0056169.

35. Ferrer I, Quintanal-Villalonga A, Molina-Pinelo S, Garcia-Heredia JM, Perez M, Suarez R, et al. MAP17 predicts sensitivity to platinum-based therapy, EGFR inhibitors and the proteasome inhibitor bortezomib in lung adenocarcinoma. J Exp Clin Cancer Res. 2018;37:195.

36. Chen X, Liao Y, Yu Y, Zhu P, Li J, Qin L, et al. Elevation of MAP17 enhances the malignant behavior of cells via the Akt/mTOR pathway in hepatocellular 
carcinoma. Oncotarget. 2017;8(54):92589-603. https://doi.org/10.18632/ oncotarget.21506.

37. Noh M, Yeo H, Ko J, Kim HK, Lee CH. MAP17 is associated with the T-helper cell cytokine-induced down-regulation of filaggrin transcription in human keratinocytes. Exp Dermatol. 2010;19(4):355-62. https://doi.org/10.1111/j.1 600-0625.2009.00902.x.

38. Guijarro MV, Link W, Rosado A, Leal JF, Carnero A. MAP17 inhibits Mycinduced apoptosis through PI3K/AKT pathway activation. Carcinogenesis. 2007;28(12):2443-50. https://doi.org/10.1093/carcin/bgm154.

39. Guijarro MV, Castro ME, Romero L, Moneo V, Carnero A. Large scale genetic screen identifies MAP17 as protein bypassing TNF-induced growth arrest. J Cell Biochem. 2007;101(1):112-21. https://doi.org/10.1002/jcb.21163.

40. Munoz-Galvan S, Gutierrez G, Perez M, Carnero A. MAP17 (PDZKIP1) expression determines sensitivity to the proteasomal inhibitor Bortezomib by preventing Cytoprotective autophagy and NFkappaB activation in breast Cancer. Mol Cancer Ther. 2015;14(6):1454-65. https://doi.org/10.1158/15357163.MCT-14-1053.

41. Perez M, Garcia-Heredia JM, Felipe-Abrio B, Munoz-Galvan S, Martin-Broto J, Carnero A. Sarcoma stratification by combined pH2AX and MAP17 (PDZK1IP1) levels for a better outcome on doxorubicin plus olaparib treatment. Signal Transduct Target Ther. 2020;5(1):195. https://doi.org/10.103 8/s41392-020-00246-z.

42. Garcia-Heredia JM, Otero-Albiol D, Perez M, Perez-Castejon E, Munoz-Galvan S, Carnero A. Breast tumor cells promotes the horizontal propagation of EMT, stemness, and metastasis by transferring the MAP17 protein between subsets of neoplastic cells. Oncogenesis. 2020;9(10):96. https://doi.org/10.103 8/541389-020-00280-0.

43. Yoshida GJ. Metabolic reprogramming: the emerging concept and associated therapeutic strategies. J Exp Clin Cancer Res. 2015;34(1):111 https://doi.org/10.1186/s13046-015-0221-y.

44. Robey RB, Hay N. Is Akt the "Warburg kinase"?-Akt-energy metabolism interactions and oncogenesis. Semin Cancer Biol. 2009;19(1):25-31. https:// doi.org/10.1016/j.semcancer.2008.11.010.

45. Al Tameemi W, Dale TP, Al-Jumaily RMK, Forsyth NR. Hypoxia-modified Cancer cell metabolism. Front Cell Dev Biol. 2019;7:4. https://doi.org/10.33 89/fcell.2019.00004.

\section{Publisher's Note}

Springer Nature remains neutral with regard to jurisdictional claims in published maps and institutional affiliations.

Ready to submit your research? Choose BMC and benefit from:

- fast, convenient online submission

- thorough peer review by experienced researchers in your field

- rapid publication on acceptance

- support for research data, including large and complex data types

- gold Open Access which fosters wider collaboration and increased citations

- maximum visibility for your research: over $100 \mathrm{M}$ website views per year

At $\mathrm{BMC}$, research is always in progress.

Learn more biomedcentral.com/submissions 